\title{
Originales
}

\section{Índice de Capacidad de Trabajo (ICT) en personal sanitario de edad avanzada e intención de jubilación anticipada}

\section{Work Ability Index in Healthcare Elderly Workers and Early Retirement Intention}

\author{
Caridad León Robles ${ }^{1}$ \\ 1. Médico especialista Medicina del Trabajo. Bizkaia. España. \\ Recibido: 06-08-2018 \\ Aceptado: 16-07-2019 \\ Correspondencia \\ Caridad León Robles \\ Correo electrónico: caridadpilar@gmail.com
}

Resumen

Se realizó un estudio analítico transversal en el que participaron de manera voluntaria trabajadores sanitarios, estatutarios de 62 a 64 años de un hospital terciario. El objetivo fue identificar la relación entre el nivel de Índice de Capacidad para el Trabajo (ICT) con sus intenciones de jubilación anticipada voluntaria. Para lo cual se aplicó el cuestionario, traducido, del ICT del Instituto Finlandés de salud ocupacional y otro cuestionario con preguntas de corte sociolaboral.

Participaron 56 trabajadores (médicos, personal de enfermería y auxiliares de enfermería). Observamos que el $100 \%$ de los que tenían un ICT deteriorado tenían intenciones de jubilarse anticipadamente (Fisher p: 0,019). Las Intenciones de jubilación anticipada fueron disminuyendo según aumentaba la edad ( $\mathrm{X}^{2}$ para tendencia línea: 4,61; $p=0,03)$. El 73\% de las auxiliares de enfermería tenían intenciones de jubilarse anticipadamente, seguido del 65\% del personal de enfermería y el 14, 2\% de médicos (Fisher p: 0,019). Más del $50 \%$ de los trabajadores que pretendían una jubilación anticipada trabajaban a turno rotatorio y de estos el 79\% tenía intenciones de jubilación anticipada a diferencia de otros turnos (Fisher p 0,048). El trabajo en turno rotatorio comprende un riesgo tres veces mayor de plantearse una jubilación anticipada que el resto de turnos (OR: 3,8; $95 \%$ CI: 1,13- 12,6; $p=0,0298$ ).

Med Segur Trab (Internet). 2019;65(254):49-58

Palabras clave: Índice de capacidad para el trabajo, trabajador de mayor edad, jubilación anticipada, trabajo a turnos.

Abstract

A cross-sectional analytical study was carried out in which health workers voluntarily participated, these were statutory staff from 62 to 64 years from a tertiary referral hospital.

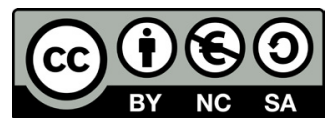

https://creativecommons.org/licenses/by-nc-sa/4.0/ 
Objective: To identify the relationship between the Level of Work Ability Index (WAI) with their voluntary early retirement intentions. To this end, the questionnaire, translated from the WAI of the Finnish Institute of Occupational Health together with another questionnaire with socio-labor questions, was applied. Results: 56 workers participated (physicians, nursing assistants and nurses). We found out that $100 \%$ of those with an impaired WAI had the intention to retire early (Fisher p: 0.019). The Early Retirement Intentions were decreasing as the age increased (X2 for trend line: 4.61, p = 0.03). 73\% of nursing assistants followed by $65 \%$ of nurses and 14. $2 \%$ of physicians (Fisher p: 0.019) intended to retire early. More than $50 \%$ of the workers who had intentions of early retirement worked on a rotating shift and $79 \%$ of them had intentions of early retirement unlike other shifts (Fisher p 0.048). Swift work has 3 times more risk of considering early retirement than the rest of the shifts (OR: 3.8, 95\% CI: 1.13-12.6, $\mathrm{p}=0.0298$ ).

Med Segur Trab (Internet). 2019;65(254):49-58

Keywords: Older workers, Work Ability Index, health worker, health professionals, elderly workers, early retirement, swift work. 


\section{INTRODUCCIÓN}

Existen previsiones que apuntan a dos factores que afectarán la estructura de la mano de obra en la Unión Europea (UE) para el año 2030: por un lado, la generación del "Baby Boom" alcanzará la edad de jubilación y, por otro, la proporción de población en edad activa será baja y con poca capacidad de renovación debido a la baja natalidad de la población de esta zona Euro ${ }^{1}$. En España, las cifras de INE (Instituto Nacional de Estadística) muestran que la distribución de la edad de los médicos en España es aproximadamente un 35,9\% de menores de 45 años, 49,6\% se encuentran entre 45 y 64 y el 14,5\% son mayores de 64 años ${ }^{2}$. El estudio del Colegio Oficial de Médicos sobre la Situación Laboral de Médicos en España encuentra que los médicos en edad de 45-60 años se concentran en los Hospitales de mayor complejidad $^{3}$. El Ministerio de Sanidad y Consumo revela que en Atención Primaria, incluyendo Medicina de Familia y Pediatría, aproximadamente el 34\% y el 40\% respectivamente son mayores de 50 años ${ }^{4}$. En contraposición, estudios realizados en la Unión Europea describen una tendencia a la salida prematura del campo laboral de los trabajadores de mayor edad 5 .

Tuomi, Ilmarinen y colaboradores investigadores del Instituto de Salud Ocupacional de Finlandia (FIOH) entre las décadas de los 80 y los 90, encontraron que el retiro anticipado de la vida laboral principalmente por muerte e incapacidad laboral tenía como factor pronóstico la disminución de la Capacidad para el trabajo. Ellos conceptuaron la Capacidad para el Trabajo como la medida en que un trabajador podía desempeñar su labor atendiendo a las demandas específicas de las tareas, su salud individual y sus recursos mentales ${ }^{6,7,8}$. Actualmente, la capacidad para el Trabajo se considera un concepto abstracto, ya que no solo se asocia a la situación de salud física y competencias profesionales de los trabajadores, sino que contempla un bienestar psicológico, una organización laboral armónica, incluso otros factores que rebasan el entorno laboral propiamente, como son los estilos y condiciones de vida ${ }^{9,10}$. Ésta se mide a través de un cuestionario auto-administrado que permite cuantificar el nivel de capacidad de trabajo individual al que se ha llamado Índice de Capacidad para el Trabajo (ICT). El FIOH es pionero en la aplicación de este método y recomienda su uso en los exámenes de salud periódicos de los trabajadores ${ }^{7}$. Su uso se ha extendido y ha sido validado y traducido a varios idiomas incluyendo el español ${ }^{10,11}$. Este Índice es un indicador amplio de la capacidad para el trabajo basada en la percepción del trabajador sobre sus recursos personales y las demandas del trabajo y disminuye en aproximadamente el 30\% en los mayores de 45 años y varían según el sector económico en el que se mida ${ }^{8}$ dato importante sabiendo que la disminución de la Capacidad para el trabajo irá asociada a una mayor probabilidad de jubilación anticipada.

En salud ocupacional el ICT es de especial relevancia debido a que se le atribuye el poder de predecir la incapacidad permanente en el futuro y el retiro anticipado del trabajo ${ }^{6,8,9}$. Este último también ha sido relacionado a otros factores como la falta de reconocimiento laboral, la baja satisfacción y motivación para el trabajo, así como sentirse estresado y percibir una desfavorable relación entre el esfuerzo y las gratificaciones ${ }^{12}$. Así también se mencionan otros factores como el nivel educativo, estado civil, ingresos salariales o las relaciones intrafamiliares y apoyo social ${ }^{12,13,14,15}$, expectativas personales y de futuro, el reconocimiento social y la satisfacción con el trabajo, el clima laboral y la organización del trabajo ${ }^{14,16,17}$.

Es así que la finalidad de este estudio es determinar si los niveles de ICT bajo o moderado, durante el periodo de transición hacia la jubilación en personal sanitario mayor de 62 a 64 años, se relacionan con la presencia de intenciones de Jubilación Anticipada. De esta forma, valorar la necesidad de implementar actividades preventivas con miras a asegurar el bienestar de este trabajador durante el resto de su etapa productiva, intentando evitar su retiro del campo laboral de forma anticipada. 


\section{METODOLOGÍA}

Se realizó un estudio analítico transversal en el que participaron Médicos, Enfermeras y Auxiliares de Enfermería de entre 62-64 años que trabajaban en un hospital terciario. Para desarrollar este trabajo se solicitaron los permisos a la dirección del Hospital recursos Humanos y el visto bueno del comité de ética.

Tras recibir el listado de trabajadores del hospital, se seleccionaron a los trabajadores que cumplían los criterios de inclusión tales como la edad, relación laboral estatutaria y personal asistencial con antigüedad de al menos un año en el servicio. No se incluyeron en el estudio aquellos trabajadores que no se encontraban activos en su servicio durante el período de recogida de datos (Incapacidad temporal, licencia, permiso, vacaciones...) ni aquellos que no quisieron participar en el estudio.

Para la recogida de datos se utilizó la versión corta del cuestionario del ICT de la FIOH en su versión validada y traducida al español ${ }^{10,11}$. La dimensión sobre los recursos mentales fue simplificada en una pregunta única. También se utilizó un cuestionario de características sociodemográficas, que contenía la pregunta directa sobre las intenciones de jubilación anticipada. En la recolección de los datos se enfatizó en asegurar el anonimato y la confidencialidad de los datos.

Ambos cuestionarios se pilotaron en 10 trabajadores de entre 55 a 64 años, escogidos por cercanía. Tras lo cual se hicieron algunas modificaciones de expresión en dicho cuestionario adaptándolo al lenguaje cotidiano de la zona, manteniendo en todo momento el sentido de la pregunta.

El análisis de datos se desarrolló utilizando las herramientas de cálculo del Microsoft Excel y el Programa SPSS versión 24.0. Para conocer la frecuencia de intenciones de Jubilación Anticipada y los niveles de ICT, se elaboraron gráficos y tablas de frecuencias y para la estadística Inferencial sobre la relación entre las variables sociolaborales y nivel de ICT con las intenciones de jubilación anticipada, se aplicaron según necesidad la Prueba exacta de Fisher, $\mathrm{X}^{2}$ para tendencia línea y en los casos que la tabla de contingencia lo permitía medidas de Fuerza de asociación (Odds ratio).

\section{RESULTADOS}

Obtuvimos un total de 56 trabajadores que contestaron a los cuestionarios. La tasa de respuesta fue menor del 30\% del total de trabajadores que cumplían los criterios de inclusión. De la población total que contestó los cuestionarios encontramos que solo 10 trabajadores eran del sexo masculino; el 82\% restante eran mujeres. En relación a las características sociodemográficas, la distribución por edades fue de 21 trabajadores de 62 años, 21 trabajadores de 63 años y 14 trabajadores de 64 años. El 60\% (n: 34) de estos se encontraban en una relación de pareja, el $20 \%$ sin pareja y el resto en otras situaciones. El 41\% (n: 23) tenía un familiar que dependía de él/ella económicamente y/o de cuidado. En cuanto a las características laborales, el 46,43\% eran Auxiliares de Enfermería, seguido por $42,07 \%$ de Licenciadas en enfermería y 12,5\% de médicos (n: 5). El área de especialidades Médicas fue la más representativa con un 61\%, seguida del área Quirúrgica con otro 34\% y el resto Urgencias generales. El 89\% tenía una antigüedad en el puesto de más de 2 años (n: 50). Por último, el 52\% se encontraba trabajando en turno mañana, y el resto estaba en algún tipo de prolongación de jornada o turno rotatorio. Este último fue el turno más frecuente entre las Auxiliares de Enfermería. Debido al escaso número de médicos que respondieron la encuesta, la variable prolongación de Jornada/guardia no aportó datos relevantes.

Los niveles de ICT de más del $80 \%$ de los datos obtenidos se encontraban entre Excelente y Bueno y solo 2 casos de ICT bajo. Reagrupamos los valores de ICT en "Niveles Deteriorados" donde se incluye Bajo y Moderado y "Niveles Satisfactorios" en los que se agrupa los ICT Buenos y Excelentes (Gráfico 1). 
Gráfico 1. Distribución de los trabajadores sanitarios según nivel de ICT.

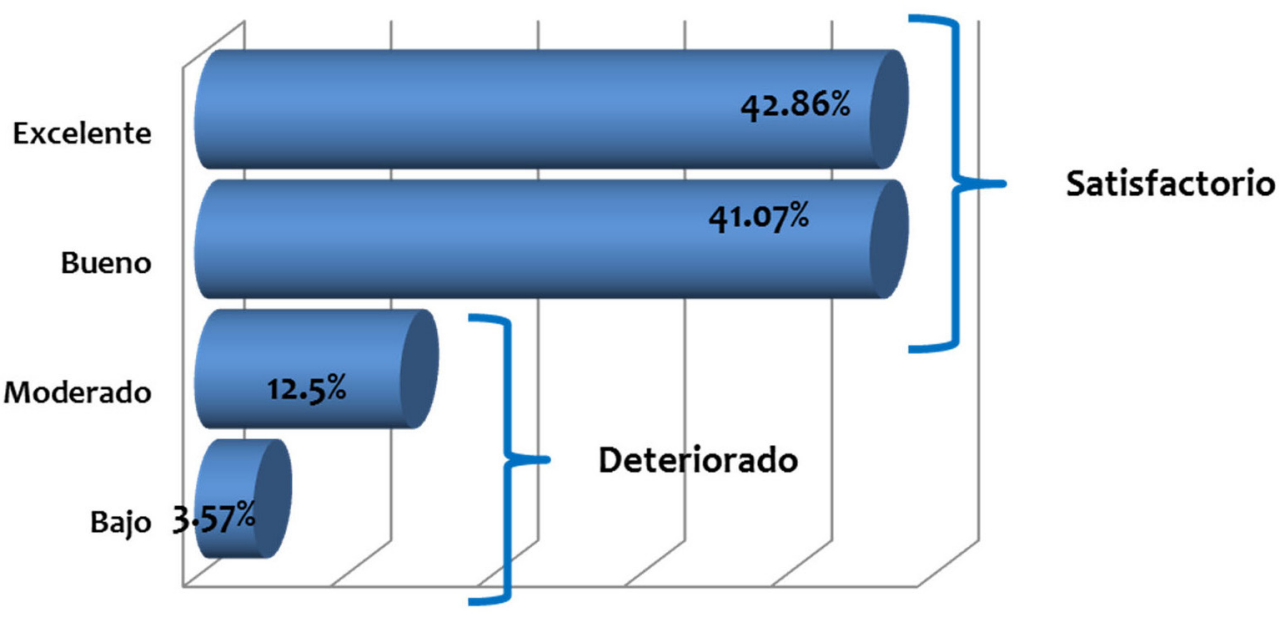

La presencia de Intenciones de Jubilación Anticipada fue del 62,5\%.

Gráfico 2. Distribución de los trabajadores sanitarios según intenciones de jubilación anticipada.

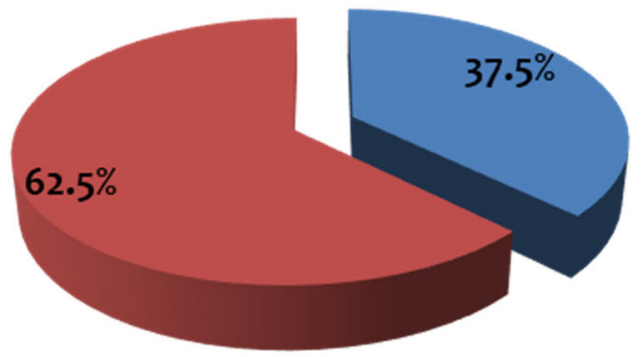

En relación a la estadística diferencial, se encontró que el 55\% de los que trabajan en áreas médicas tenían valores de ICT satisfactorios. Lo que se correspondía con una $p$ significativa (Test de Fisher: p: 0.042) No se encontró relación alguna u asociación entre los Niveles de ICT con el resto de variables sociolaborales estudiadas.

En la "tabla 1" el 100\% de los que tenían un "ICT deteriorado" tenían intenciones de jubilarse a diferencia de los que tenían niveles satisfactorios de ICT (55,3\%), resultados que fueron estadísticamente significativos. No pudimos determinar la fuerza de asociación debido a la existencia de un valor de cero en la tabla de contingencia.

Tabla 1. Nivel de ICT e intenciones de jubilación anticipada.

\begin{tabular}{l|c|c|c|c}
\hline \multirow{2}{*}{\multicolumn{1}{c|}{ Nivel ICT }} & \multicolumn{4}{c}{ Intención de Jubilación Anticipada Voluntaria } \\
\cline { 2 - 5 } & Sí & No & \multicolumn{2}{c}{ Total } \\
\cline { 2 - 5 } & $\mathbf{n}$ & $\mathbf{n}$ & $\mathbf{n}$ & $\%$ \\
\hline Deteriorado & 9 & 0 & 9 & 100,0 \\
\hline Satisfactorio & 26 & 21 & 47 & 55,3 \\
\hline Total & $\mathbf{3 5}$ & $\mathbf{2 1}$ & $\mathbf{5 6}$ & \\
\hline $\mathbf{p = 0 , 0 1 9}$ & \multicolumn{5}{|l}{} \\
\hline
\end{tabular}

En la tabla 2, se analiza la prevalencia de intención de jubilación anticipada, estratificada por las categorías de otras variables de interés. Como se observa en el análisis bivariante, la presencia de intenciones de Jubilación anticipada fue disminuyendo con la edad evidenciando una tendencia lineal estadísticamente significativa. Por otra parte, esta prevalencia no se distribuyó homogéneamente según las diferentes categorías 
profesionales $(\mathrm{p}=0,01)$, siendo mayor la intención de jubilación anticipada entre Auxiliares de Enfermería (73\%), seguidas por Enfermería (65\%). La prevalencia entre el personal médico fue del $14,2 \%$.

Tabla 2. Intenciones de jubilación anticipada y variables sociolaborales.

\begin{tabular}{|c|c|c|c|c|c|c|}
\hline \multirow{2}{*}{\multicolumn{2}{|c|}{$\begin{array}{c}\text { Variables } \\
\text { socio laborales }\end{array}$}} & \multicolumn{3}{|c|}{$\begin{array}{c}\text { Intenciones de } \\
\text { jubilación anticipada }\end{array}$} & \multirow{3}{*}{$\begin{array}{c}\begin{array}{c}\text { Presencia de } \\
\text { intenciones } \\
\text { de jubilación } \\
\text { anticipada (\%) }\end{array} \\
67.4\end{array}$} & \multirow{2}{*}{ Valor $p$ estimado } \\
\hline & & \multirow{2}{*}{$\begin{array}{l}\text { Sí } \\
\mathbf{n} \\
31\end{array}$} & \multirow{2}{*}{$\begin{array}{c}\text { No } \\
\text { n } \\
15\end{array}$} & \multirow{2}{*}{$\frac{n}{46}$} & & \\
\hline \multirow{2}{*}{$\begin{array}{l}\text { Identidad } \\
\text { de Género }\end{array}$} & Femenino & & & & & \multirow{2}{*}{$\mathrm{p}=0,1517$} \\
\hline & Masculino & 4 & 6 & 10 & 40 & \\
\hline \multirow{3}{*}{ Edad } & 62 & 16 & 5 & 21 & 76,2 & \multirow{3}{*}{$\mathrm{p}($ tendencia lineal $)=0,03$} \\
\hline & 63 & 13 & 8 & 21 & 61,9 & \\
\hline & 64 & 6 & 8 & 14 & 42,9 & \\
\hline \multirow{3}{*}{$\begin{array}{l}\text { Situación } \\
\text { de Pareja }\end{array}$} & En pareja & 20 & 14 & 34 & 58.8 & \multirow{3}{*}{$\mathrm{p}=0,801$} \\
\hline & Sin pareja & 8 & 3 & 11 & 72.7 & \\
\hline & Otros & 7 & 4 & 11 & 63.6 & \\
\hline \multirow{2}{*}{$\begin{array}{l}\text { Familiar en } \\
\text { Situación de } \\
\text { Dependencia }\end{array}$} & Sí & 14 & 9 & 23 & 60,8 & \multirow{2}{*}{$\mathrm{p}=0,833$} \\
\hline & No & 21 & 12 & 33 & 63,6 & \\
\hline \multirow{3}{*}{$\begin{array}{l}\text { Categoría } \\
\text { Profesional }\end{array}$} & $\begin{array}{l}\text { Auxiliar de } \\
\text { Enfermería }\end{array}$ & 19 & 7 & 26 & 73 & \multirow{3}{*}{$\mathrm{p}=0,019$} \\
\hline & Enfermería & 15 & 8 & 23 & 65,2 & \\
\hline & Médico & 1 & 6 & 7 & 14,2 & \\
\hline \multirow{3}{*}{$\begin{array}{l}\text { Área de } \\
\text { Trabajo }\end{array}$} & Médicas & 19 & 15 & 34 & 55,8 & \multirow{3}{*}{$\mathrm{p}=0,391$} \\
\hline & Quirúrgicas & 14 & 5 & 19 & 73,7 & \\
\hline & Urgencias & 2 & 1 & 3 & 66,7 & \\
\hline \multirow{2}{*}{$\begin{array}{l}\text { Antigüedad } \\
\text { Puesto }\end{array}$} & 2 años a más & 31 & 19 & 50 & 62 & \multirow{2}{*}{$\mathrm{p}=1,00$} \\
\hline & Menos de 2 años & 4 & 2 & 6 & 66,7 & \\
\hline \multirow[b]{2}{*}{ Turnicidad } & Rotatorio & 19 & 5 & 24 & 79,2 & \multirow{2}{*}{$\begin{array}{c}\mathrm{p}=0,029 \\
\text { OR: } 3,8(\mathrm{IC} 95 \%: 1,13-12,67)\end{array}$} \\
\hline & $\begin{array}{c}\text { Mañana/tarde } \\
\text { y mañana }\end{array}$ & 16 & 16 & 32 & 50 & \\
\hline \multicolumn{2}{|l|}{ Total } & 35 & 21 & 56 & 62,5 & \\
\hline
\end{tabular}

Por último, el trabajo en turnos rotatorios incrementó el porcentaje de trabajadores que expresaron su intención de jubilarse anticipadamente, estimándose dicha frecuencia en un $79 \%$.

Así mismo, encontramos que el turno rotatorio tiene un riesgo de 3 veces más de plantearse la jubilación anticipada que el resto de turnos (tabla 2).

\section{DISCUSIÓN}

El hecho de que la mayor proporción de trabajadores participantes fuera del sexo femenino es un dato que se corresponde con la conocida feminización del personal sanitari0, la cual llega a más del $75 \%^{18}$. En el servicio Vasco de Salud, el porcentaje llega aproximadamente al $\mathbf{9 0 \%}$ entre las categorías profesionales de Enfermería y Auxiliar de Enfermería $^{19}$.

Por otro lado, identificamos que el 16\% de los trabajadores que contestaron a nuestro cuestionario de ICT, obtuvieron niveles de ICT deteriorados (bajo y moderado). Sabiendo 
que la relevancia del ICT radica en que se ha demostrado que los niveles de ICT deteriorados de un individuo se pueden mejorar y restablecer tras intervenciones específicas $^{6,20}$. Esto nos podría sugerir que perfiles similares a los de este estudio podrían requerir de intervención específica, con miras a mejorar los niveles de ICT. Además, algunos estudios le atribuyen la posibilidad de predecir la vida activa y significativa después de la jubilación, así como identificar los tipos de trabajo que causan problemas a los "trabajadores mayores" 2 . El ICT como tal no califica una incapacidad laboral sino una incongruencia actual y futura entre las exigencias del trabajo y la capacidad para trabajar.

A diferencia de lo referido por la literatura que menciona una correlación negativa entre la edad y el nivel de ICT ${ }^{22,23}$, se encontró que más del 80\% de nuestros trabajadores tenía niveles de ICT entre Bueno y Excelente. Tal y como concluye Van der Berg et al., a través de una revisión sistemática sobre los factores determinantes del ICT, la edad no es un factor determinante en los niveles de ICT, por tanto, no encuentra relación directamente proporcional con la edad ${ }^{24}$. Padula et al., en un estudio comparativo en el que busca diferencias entre trabajadores mayores de 50 años y trabajadores jóvenes que se desempeñaban en diversos sectores económicos, encuentra que los trabajadores mayores tienden a usar más medicamentos, tienen también más diagnósticos de enfermedades e indicadores de sobrepeso y obesidad fuera de rangos recomendados. Pero, en cambio, no se evidencian diferencias estadísticamente significativas en los niveles de (ICT) encontrados en ambos grupos ${ }^{25}$. De la misma manera, un estudio realizado en Suecia con más de 2000 trabajadores sociosanitarios, cuya media de edad era 47 años, determina que el 19\% de los trabajadores tenían ICT entre bajo y moderado ${ }^{22}$. Pese a que existen estudios que no han relacionado directamente la edad con los niveles de ICT, tenemos en cuenta que en este estudio puede existir un sesgo de supervivencia del trabajador sano y que ello responda a la alta prevalencia de niveles de ICT encontrados en nuestro estudio. Otro factor que hay que tener en cuenta es el "efecto voluntario sano", que implica que las personas sanas son más propensas a participar en las encuestas de salud que los trabajadores que experimentan problemas de salud ${ }^{26}$. Hecho que se hace más evidente al encontrar en nuestro análisis que la Intención de Jubilación va disminuyendo según aumenta la edad, lo que deja entrever el sesgo de supervivencia del trabajador sano. También podría haber influenciado el hecho de que la mayor proporción de trabajadores eran del sexo femenino y, en este sentido, los principales investigadores del ICT mencionan que, en mayores de 60 años, se les atribuye mejores niveles de ICT al sexo femenino frente a los hombres ${ }^{27}$.

Consideramos que el uso de la versión corta del ICT (sin el listado de enfermedades), no ha tenido influencia importante en la discriminación de los niveles de ICT, ya que existen varios estudios que validan el score corto (WAS) que solo incluye la primera pregunta del ICT, y le atribuyen el mismo poder predictivo a largo plazo que la versión larga del ICT o WAI en inglés ${ }^{23,28}$.

Los resultados encontrados, como versan las fuentes ${ }^{23,27,29}$, dependen del grupo en el que se realizan las evaluaciones. En nuestro estudio, la mayor proporción de trabajadores fue representada por personal de enfermería, tanto Auxiliares de Enfermería (46\%) como Enfermeras (41\%), así que los factores que podrían influenciar en los niveles ICT que encontramos, podrían estar condicionados por las percepciones de estos grupos laborales. Al respecto, nosotros encontramos que los trabajadores sanitarios que trabajaban en las áreas médicas tenían la mayor proporción de niveles de ICT satisfactorio a diferencia de los trabajadores de áreas quirúrgicas y urgencias, tal como se menciona en un estudio realizado en enfermeras de urgencias donde sí se encontró correlación entre la edad y los niveles deteriorados de ICT $^{30}$. Esto podría estar relacionado al estilo de trabajo de las anteriormente mencionadas, que se caracteriza por altos niveles de estrés tal y como se ha encontrado en algunos estudios, cuestión que se correlaciona negativamente con el $\mathrm{ICT}^{14}$.

En relación a la pregunta directa sobre las intenciones de jubilación, el 62,5\% se jubilaría anticipadamente de forma voluntaria; sin embargo, existe la posibilidad de que 
los resultados de intenciones de jubilación anticipada sean incluso mayores. En la memoria anual del hospital se menciona que el porcentaje de diplomados sanitarios que se han jubilado voluntariamente dentro de ese año llega al 31\% del total, seguido de un 28\% de técnicas auxiliares sanitarios y de solo un $5.4 \%$ de Facultativos médicos. Esto podría responder al sesgo de "deseabilidad social" tal y como lo describió en su estudio de Corné A. et al., donde situaciones como la pensión de invalidez y el desempleo son valoradas negativamente ${ }^{31}$.

En nuestro estudio, las intenciones de jubilación se relacionaron significativamente con los niveles deteriorados de ICT según lo menciona la literatura ${ }^{27,32,33}$. Aunque en el caso de Fernández J. no encontró relación entre la salud percibida y las intenciones de prejubilación en personal sanitario ${ }^{34}$. Además, encontramos asociación entre las intenciones de jubilación y el trabajo a turnos, ya que éstos tienen un riesgo de hasta tres veces mayor en la intención de jubilarse anticipadamente frente a los que no trabajan de forma rotatoria.

Finalmente, la implementación de medidas preventivas podrían resultar efectivas con miras a evitar el retiro anticipado. AGEM de Boer et., al realizar un seguimiento tras intervención en trabajadores con ICT bajos y moderados, concluye que tras dos años de implementación del programa, fue ligeramente efectivo en la mejora de la capacidad de trabajo, pero no en la reducción de las pensiones de invalidez. Al mismo tiempo, el programa recomienda que los trabajadores con un alto riesgo para la pensión por invalidez podrían necesitar una intervención específica y multidisciplinar más ampliada ${ }^{32}$. Advirson et al., en su estudio de seguimiento por dos años, tras la implementación de medidas con el fin de mejorar los niveles de ICT de su trabajadores, encontró que el ascenso a una categoría estaba asociada a la actividad física frente al sedentarismo ${ }^{22}$.

\section{LIMITACIONES Y SESGOS}

Las principales limitaciones de este estudio fueron la falta de recursos para la etapa de recogida de datos, así como la idiosincrasia del personal sanitaria reflejada en la escasa participación del personal médico, lo que podría condicionar algunos de los resultados encontrados. Por otro lado, se perdieron datos debido a la dificultad de coincidir con los trabajadores a turnos.

Una de las principales limitaciones del estudio es que la muestra es pequeña, por tanto los resultados del mismo no puedan ser extrapolados a otras poblaciones ni al total de los trabajadores sanitarios del hospital.

Por último existen sesgos importantes que pueden afectar los resultados de este trabajo, tales como el de la supervivencia del trabajador sano y el «efecto voluntario sano" evidentes.

\section{CONCLUSIONES}

- Existe una mayor proporción en la intención de jubilación anticipada en los trabajadores sanitarios, estatutarios, de 62 a 64 años que tienen niveles de ICT deteriorados (Moderado o Bajo) frente a los trabajadores con niveles de ICT satisfactorio ( Bueno o Excelente).

- El $\mathbf{1 0 0 \%}$ de los trabajadores que tuvieron niveles de ICT deteriorados tenían intenciones de Jubilación anticipada frente al 55\% de los trabajadores con niveles de ICT satisfactorio.

- El 62,5\% de los trabajadores tienen intenciones de Jubilarse Anticipadamente de forma voluntaria.

- Aproximadamente el 84\% de los trabajadores sanitarios tienen niveles de ICT entre buenos y excelentes (Satisfactorios), seguidos por el 16\% con niveles de ICT Deteriorados (12,5\% nivel Moderado y 3,5\% Bajo). 
- Los trabajadores sanitarios cuyos puestos se desempeñan en áreas médicas tienen mejores niveles de ICT que sus homólogos que trabajan en áreas quirúrgicas o de urgencias.

- En la categoría de Auxiliares de Enfermería hay una mayor proporción en la Intención de Jubilación anticipada frente a las otras categorías profesionales del estudio, como Personal de enfermería o Facultativos Médicos.

- La turnicidad se asocia con un riesgo de hasta tres veces más en la intención de jubilación anticipada frente al trabajo de turno mañana o mañana/tarde.

- La Intención de Jubilación anticipada de los trabajadores sanitarios va disminuyendo según aumenta la edad.

- Sería adecuado establecer la valoración temprana del ICT como un instrumento de gestión de la edad en personal sanitario de mayor edad para poder enfocar acciones específicas, si se requieren, con miras a asegurar el bienestar de este grupo durante el resto de su etapa productiva, intentando evitar su retiro laboral de forma anticipada.

\section{AGRADECIMIENTOS}

A todas las personas que de forma directa o indirecta han ayudado o aportado en esta investigación y especialmente a Esperanza Álvarez Maeztu y Marta Zimmermann Verdejo por sus importantes aportes en mi investigación y a Amelia Sáez Turrientes por su valiosa colaboración en la recolección de datos.

\section{REFERENCIAS BILIOGRÁFICAS}

1. Morschauser, M. Sochert, R. Trabajo Saludable en una Europa que Envejece Estrategias e Instrumentos para Prolongar la Vida Laboral [en línea] Disponible en: http://www.ageingatwork.eu/resources/healthwork-in-an-ageing-europe-enwhp-3.pdf [Consulta: 14 Julio 2015]

2. INE. Estadística de Profesionales Sanitarios Colegiados Año 2015 [en línea] Disponible en: http://www. ine.es/prensa/np970.pdf [Consulta: 14 Julio 2016]

3. Estudio sobre la Situación Laboral de Médicos en España. Tercera Oleada. [en línea] Disponible en: https://www.cgcom.es/sites/default/files/u183/InformeResultadosTercerMuestreoEncuestaSituaci\%C3\%B 3nLaboraldelosM\%C3\%A9dicosenEspa\%C3\%B1a30demarzode2016.pdf [Consulta: 14 Julio 2016]

4. Ministerio De Sanidad, Servicios Sociales E Igualdad. Informe Anual Del Sistema Nacional De Salud [En Línea] Disponible en: https://www.mscbs.gob.es/estadEstudios/estadisticas/sisInfSanSNS/tablas Estadisticas/Inf_Anual_SNS_2015.1.pdf [Consulta: 14 Julio 2016]

5. Roelen, C et al. Work ability index as tool to identify workers at risk of premature work exit. Journal of occupational rehabilitation. 2014: 24(4), 747-754.

6. Tuomi K. Finnish research project on aging workers in 1981-1 992 Scand J Work Environ Health 1997;23(suppl 1):7-11

7. Gould R et al. 2008. Dimensions of Work Ability [Internet]. Helsinsky. Finnish Institute of Occupational Health [Citado 10 Setiembre 2015]. Disponible en: https://sivusto.kykyviisari.fi/wp-content/uploads/ 2018/09/Gould-Dimensions-of-work-ability.pdf

8. Ilmarinen J. Promoción del envejecimiento activo en el trabajo. [en línea] Disponible en: https://osha. europa.eu/es/tools-and-publications/publications/articles/promoting-active-ageing-in-the-workplace [Consulta: 14 Julio 2015]

9. Ilmarinen J, Tuomi K, Seitsamo J. New dimensions of work ability. International Congress Series, June 2005, 1286 Pages 3-7

10. López GM, del Castillo NP, Oramas A. Validez y Confiabilidad del Cuestionario Índice de Capacidad de Trabajo (ICT) en su Versión Cubana. Revista Cubana de Salud y Trabajo 2011;12(2):29-34

11. Peralta, $\mathrm{N}$ et al. Validez y confiabilidad del Índice de Capacidad para el Trabajo en trabajadores del primer nivel de atención de salud en Argentina. Salud Colectiva. 2012;8(2):163-173 [en línea]. Disponible en: https://www.scielosp.org/article/ssm/content/raw/?resource_ssm_path=/media/assets/scol/v8n2/ v8n2a05.pdf [Consulta: 24 setiembre 2015] 
12. López GM, et al. Motivaciones relacionadas con la Intención de Jubilación en Trabajadores de la Salud de Segundo Nivel de Atención. Revista Cubana de Salud y Trabajo. 2015;16(2):25-30 [en línea] Disponible en: https://www.medigraphic.com/pdfs/revcubsaltra/cst-2015/cst152d.pdf [Consulta: 14 Julio 2015]

13. P. Suadicani, J. P. Bonde, K. Olesen and F. Gyntelberg. Job satisfaction and intention to quit the job. Occupational Medicine 2013;63:96-102

14. Golubic R. et al. Work-related stress, education and work ability among hospital nurses. JAN. 2009; 65 (10) 2056-2066

15. K. Henkens, M. Leenders. Burnout e intenciones de jubilación anticipada entre empleados mayores. Rev Psicol Trab Organ. 2008; 24(3)

16. Heponiemi T, Kouvonen A, Vänskä J, Halila H, Sinervo T, Kivimäki M, Elovainio M. Health, psychosocial factors and retirement intentions among Finnish physicians. Occup Med (Lond). 2008 Sep;58(6):406-12

17. Alavinia SM, de Boer AG, van Duivenbooden C, Frings-Dresen MH, Burdorf A. Determinants of work ability and its predictive value for disability. Occup Med 2009; 59(1):32-7.

18. P. Vázquez Vega. La Feminización de las profesiones sanitarias. Fundación BBva. Bilbao 2010.

19. Departamento de Salud. Gobierno Vasco. Memoria Osakidetza 2016 [En Línea] Disponible En: http:// www.osakidetza.euskadi.eus/contenidos/informacion/osk_publicaciones/es_publi/adjuntos/memorias/ Memoria_2016_es.pdf

20. J. Ilmarinen Snel, R. Cremer (Eds.), Work and aging: a European prospective (1994), Amsterdam: Taylor \& Francis, pp. 197-211

21. Tony Maltby Extending Working Lives? Employability, Work Ability and Better Quality Working Lives . Social Policy \& Society 10:3, 299-308. Cambridge University Press 2011

22. Arvidson $\mathrm{E}$ et al The level of leisure time physical activity is associated with work ability-a cross sectional and prospective study of health care workers. BMC Public Health. 2013 Sep 17;13:855

23. El Fassi M. et al Work ability assessment in a worker population: comparison and determinants of Work Ability index and Work Ability score. BMC Public Health. 2013. 8;13:305

24. Van den Berg, TIJ, Elders, LAM, De Zwart, BCH. The effects of work-related and individual factors on the Work Ability Index: A systematic review. Occup Environ Med 2009;66:211-20

25. Padula RS, Comper ML, Moraes SA, Sabbagh C, Pagliato WJ, Perracini MR. The work ability index and functional capacity among older workers. Braz J Phys Ther 2013 Jul;17(4):382-91.

26. Bethencourt A, Prieto M. Relationship between working incapacity and Work Ability Index usage. Med Segur Trab (Internet) 2016; 62 (242) 66-78

27. Ilmarinen J. The ageing workforce-challenges for occupational health. Occup Med (Lond). 2006; 56(6):362-4.

28. Schouten LS et al. Shortened version of the work ability index to identify workers at risk of long-term sickness absence. Eur J Public Health. 2016; 26(2):301-5.

29. Suleiman A. Occupational safety and heath professionals work ability concept perception and comprehension of regulations. Work. 2017;56(3):483-490

30. Duran E, Monteiro M, Para O. Trabalho Entre Alho Entre Trabalhadores De Enfermagem Do ProntoSocorro De Um Hospital Universitário. Rev Latino-am Enfermagem 2004 janeiro-fevereiro; 12(1):43-9

31. Corné AM Roelen, Martijn W Heymans. Work Ability Index as Tool to Identify Workers at Risk of Premature Work Exit. J Occup Rehabil (2014) 24:747-754.

32. Soares J. Salla S, Salmaso C. Factors associates with ability in the elderly: Systematic review. Rev Bras Epidemiol. 2014 Dec;17(4):830-41.

33. Camerino D. Low-perceived work ability, ageing and intention to leave nursing: a comparison among 10 European countries. J Adv Nurs. 2006 Dec;56(5):542-52.

34. Fernández J. Los trabajadores de mayor edad: estrategias para favorecer el mantenimiento de su capacidad laboral y su intención de mantenerse laboralmente activos [en línea] Disponible en: https://docplayer. es/10802340-Jordi-fernandez-castro-investigador-principal-universidad-autonoma-de-barcelona-enerode-2008-mayo-de-2010-resumen.html [Consulta: 14 Julio 2015]

35. AGEM de Boer, A Burdorf, C van Duivenbooden, MHW Frings-Dresen. The effect of individual counselling and education on work ability and disability pension: a prospective intervention study in the construction industry. Occup Environ Med 2007; 64: 792-797

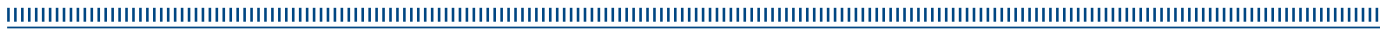

